\title{
Comparative analysis of twelve mitogenomes of Caliscelidae (Hemiptera: Fulgoromorpha) and their phylogenetic implications
}

\author{
Nian Gong ${ }^{1,2,3}$, Lin Yang ${ }^{1,2,3}$, Xiangsheng Chen ${ }^{\text {Corresp. 1, 2, } 3}$ \\ ${ }^{1}$ Guizhou University, Institute of Entomology, Guiyang, Guizhou, China \\ 2 Guizhou University, The Provincial Special Key Laboratory for Development and Utilization of Insect Resources, Guiyang, Guizhou, China \\ 3 Guizhou University, The Provincial Key Laboratory for Agricultural Pest Management of Mountainous Regions, Guiyang, Guizhou, China \\ Corresponding Author: Xiangsheng Chen \\ Email address: chenxs3218@163.com
}

Here, the complete mitochondrial genomes (mitogenomes) of 12 Caliscelidae species, Augilina tetraina , Augilina triaina, Symplana brevistrata, Symplana lii, Neosymplana vittatum, Pseudosymplanella nigrifasciata, Symplanella brevicephala, Symplanella unipuncta, Augilodes binghami, Cylindratus longicephalus, Caliscelis shandongensis, and Peltonotellus sp., were determined and comparatively analyzed. The genomes varied from 15,424 to 16,746 bp in size, comprising 37 mitochondrial genes and an A+T-rich region. The typical gene content and arrangement were similar to those of most Fulgoroidea species. The nucleotide compositions of the mitogenomes were biased toward $A / T$. All protein-coding genes (PCGs) started with a canonical ATN or GTG codon and ended with TAN or an incomplete stop codon, single T. Among 13 PCGs in 16 reported Caliscelidae mitogenomes, cox1 and atp8 show ed the lowest and highest nucleotide diversity, respectively. All PCGs evolved under purifying selection, with atp8 considered a comparatively fast-evolving gene. Phylogenetic relationships were reconstructed based on 13 PCGs in 16 Caliscelidae species and five outgroups using maximum likelihood and Bayesian inference analyses. All species of Caliscelidae formed a steadily monophyletic group with high support. Peltonotellini was present at the basal position of the phylogenetic tree. Augilini was the sister group to Caliscelini and Peltonotellini. 
1 Comparative analysis of twelve mitogenomes of Caliscelidae (Hemiptera:

\section{Fulgoromorpha) and their phylogenetic implications}

Nian Gong ${ }^{1,2,3}$, Lin Yang ${ }^{1,2,3}$, Xiang-Sheng Chen ${ }^{1,2,3}$

1'Institute of Entomology, Guizhou University, Guiyang, Guizhou, 550025, P.R. China

${ }^{2}$ The Provincial Special Key Laboratory for Development and Utilization of Insect Resources, Guizhou University, Guiyang, Guizhou, 550025, P.R. China

${ }^{3}$ The Provincial Key Laboratory for Agricultural Pest Management of Mountainous Regions, Guizhou University, Guiyang, Guizhou, 550025, P.R. China

Corresponding author: Xiang-Sheng Chen (chenxs3218@163.com)

Abstract: Here, the complete mitochondrial genomes (mitogenomes) of 12 Caliscelidae species, Augilina tetraina, Augilina triaina, Symplana brevistrata, Symplana lii, Neosymplana vittatum, Pseudosymplanella nigrifasciata, Symplanella brevicephala, Symplanella unipuncta, Augilodes binghami, Cylindratus longicephalus, Caliscelis shandongensis, and Peltonotellus sp., were determined and comparatively analyzed. The genomes varied from 15,424 to 16,746 bp in size, comprising 37 mitochondrial genes and an A+T-rich region. The typical gene content and arrangement were similar to those of most Fulgoroidea species. The nucleotide compositions of the mitogenomes were biased toward A/T. All protein-coding genes (PCGs) started with a canonical ATN or GTG codon and ended with TAN or an incomplete stop codon, single T. Among 13 PCGs in 16 reported Caliscelidae mitogenomes, coxl and atp 8 showed the lowest and highest nucleotide diversity, respectively. All PCGs evolved under purifying selection, with atp8 considered a comparatively fast-evolving gene. Phylogenetic relationships were reconstructed based on 13 PCGs in 16 Caliscelidae species and five outgroups using maximum likelihood and Bayesian inference analyses. All species of Caliscelidae formed a steadily monophyletic group 
with high support. Augilini was the sister group to Caliscelini and Peltonotellini. Caliscelinae was the sister group to Ommatidiotinae with high nodal support $(\mathrm{BPPs}=1 ; \mathrm{BPs}=100)$.

Keywords: Caliscelini, Peltonotellini, Ommatidiotinae, Mitogenome, Phylogenetics

\section{Introduction}

The family Caliscelidae (Insecta: Hemiptera: Fulgoroidea) includes a diverse group of phytophagous insects, including two subfamilies (Ommatidiotinae and Caliscelinae), five tribes (Caliscelini, Peltonotellini, Ommatidiotini, Adenissini, and Augilini), and >240 species (Bourgoin, 2021). The group is relatively small but widely distributed worldwide. Similar to other hemipteran insects, those belonging to the family Caliscelidae use piercing and sucking mouthparts to consume plant juice. Such feeding activities result in proliferation of plant cells which can further affect plant growth and development, spread plant viral diseases, and severely impact food production. Currently, most research on Caliscelidae is largely focused on the identification and description of new species; however, in a few studies, the partial mtDNA sequences of Caliscelidae species, such as Bruchomorpha beameri and Aphelonema simplex (Gwiazdowski et al., 2015; Hebert et al., 2016), have been presented. To date, among 400 hemipteran mitochondrial genomes (mitogenomes) uploaded to GenBank, only four are active caliscelid mitogenomes (Gong et al.,2021a). Considering that at least 240 caliscelid species exist, current data on caliscelid planthopper mitogenomes are extremely limited.

Insect mitogenomes are circular double-stranded DNA molecules that are maternally inherited and range from 14 to $36 \mathrm{~kb}$ in size (Cameron, 2014; Wang and Tang,2017). In metazoans, the typical mitogenome includes 37 genes, containing 2 ribosomal RNAs (rRNAs: $r r n L$ and $r r n S$ ), 13 protein-coding genes (PCGs) encoding the subunits of oxidative phosphorylation enzymes, 22 transfer RNAs (tRNAs) involved in amino acid transport, and a noncoding A+T-rich region with variable length that serves as the site of initiation for transcription and gene replication (Wolstenholme, 2017; Shadel and Clayton, 1997; Inohira et 
54

al., 1997; Boore, 1999; Osigus et al., 2013). Mitogenomes can have genome-level characteristics, such as relatively conserved gene content, base composition, modes of transcription and replication, and gene organization (Simon et al., 2006; Gissi et al., 2008). Furthermore, they are highly applicable to phylogenetic analyses in higher taxonomic categories, species identification, and population genetics (Dowton et al., 2002; Ingman et al., 2000; Ma et al., 2012).

In the present study, the mitogenomes of 12 species belonging to the family Caliscelidae were determined. All the species were collected from gramineous plants, in which their nymphs and adults are sustained by fresh plant leaves (Che et al., 2009; Chen et al., 2014; Gong et al., 2018). To further protect the quantity and quality of gramineous plants, it is necessary to determine the structure and characteristics of the mitogenomes of Caliscelidae, which is likely to provide insights into pest control strategies against these insects (Tian et al., 2001). Furthermore, determining the complete mitogenomes of the 12 studied species will enrich the Caliscelidae database for further study of Fulgoroidea phylogenetic relationships and the phylogenetic position of Caliscelidae.

In previous mitogenome-based investigations, only partial genome sequences or limited taxon sampling associated with Fulgoroidea including the Caliscelidae were conducted. Therefore, a comprehensive phylogenetic investigation into the Fulgoroidea superfamily is required based on more taxa and greater mitochondrial sequence coverage. Accordingly, we determined and comparatively analyzed the complete mitogenomes of 12 Caliscelidae species, namely, Augilina tetraina, Augilina triaina, Symplana brevistrata, Symplana lii, Neosymplana vittatum, Pseudosymplanella nigrifasciata, Symplanella brevicephala, Symplanella unipuncta, Augilodes binghami, Cylindratus longicephalus, Caliscelis shandongensis, and Peltonotellus sp., for the first time in the present study. Our analyses specifically included gene order, nucleotide composition, codon usage, tRNA secondary structure, gene overlaps, and noncoding regions. In addition, we conducted a comprehensive phylogenetic investigation on Caliscelidae with expanded mitochondrial gene data and taxon sampling. Ultimately, our aim was to use all available caliscelid mitogenomes to improve the current understanding of Caliscelidae 
81 phylogeny.

82

\section{Materials and Methods}

\section{Species collection and taxonomic identification} Specimens of Augilina tetraina $\left(24^{\circ} 70^{\prime} \mathrm{N}, 9^{\circ} 90^{\prime} \mathrm{E}\right)$, Augilina triaina $\left(21^{\circ} 58^{\prime} \mathrm{N}, 100^{\circ} 68^{\prime}\right.$ E), Symplana lii $\left(21^{\circ} 41^{\prime} \mathrm{N}, 101^{\circ} 25^{\prime} \mathrm{E}\right)$, Neosymplana vittatum $\left(24^{\circ} 69^{\prime} \mathrm{N}, 97^{\circ} 93^{\prime} \mathrm{E}\right)$, Pseudosymplanella nigrifasciata $\left(21^{\circ} 41^{\prime} \mathrm{N}, 101^{\circ} 25^{\prime} \mathrm{E}\right)$, Symplanella brevicephala $\left(21^{\circ} 41^{\prime} \mathrm{N}\right.$, $\left.101^{\circ} 25^{\prime} \mathrm{E}\right)$, and Augilodes binghami $\left(21^{\circ} 58^{\prime} \mathrm{N}, 100^{\circ} 68^{\prime} \mathrm{E}\right)$ were collected from the bamboo forest of Yunnan province in July 2019, Symplanella unipuncta $\left(18^{\circ} 41^{\prime} \mathrm{N}, 1^{\circ} 9^{\circ} 68^{\prime} \mathrm{E}\right)$ was collected from the bamboo forest of Hainan province in May 2021, Caliscelis shandongensis $\left(37^{\circ} 58^{\prime} \mathrm{N}, 118^{\circ} 68^{\prime} \mathrm{E}\right)$ was collected from phragmites of Shandong province in August 2016, Peltonotellus sp. $\left(49^{\circ} 22^{\prime} \mathrm{N}, 119^{\circ} 77^{\prime} \mathrm{E}\right)$ was collected from the grassland of Inner Mongolia province in July 2018 , and those of Symplana brevistrata $\left(25^{\circ} 25^{\prime} \mathrm{N}, 107^{\circ} 75^{\prime} \mathrm{E}\right)$ and Cylindratus longicephalus $\left(26^{\circ} 21^{\prime} \mathrm{N}, 108^{\circ} 22^{\prime} \mathrm{E}\right)$ were collected from Guizhou province in August 2019. All specimens were deposited at the Institute of Entomology, Guizhou University, Guiyang, China, where they were stored in $100 \%$ ethanol at $-20^{\circ} \mathrm{C}$ until further use. These 12 species were identified by Nian Gong according to morphological descriptions and illustrations (especially of genitalia) provided by Chen et al. (2014), Meng et al. (2015), and Gong et al. (2020).

\section{DNA extraction and sequencing}

Total genomic DNA was extracted from the muscle tissue of each Caliscelidae species using the Takara Genomic DNA Extraction Kit (Sangon Biotech, Shanghai, China). Use a dissecting needle to remove the muscle tissue of these insects. Specimens were incubated at $56^{\circ} \mathrm{C}$ for $6 \mathrm{~h}$ to lyse completely and the total genomic DNA was eluted in $50 \mathrm{ml}$ double-distill water (ddH2O), while the remaining steps were following the manufacturer's instructions. Quality of the extracted DNA was checked on 1\% agarose gel. The extracted genomic DNA was stored at $-20^{\circ} \mathrm{C}$ until further use. Voucher specimens with male genitalia and DNA samples have been 
deposited at the Institute of Entomology, Guizhou University, Guiyang, China. Complete mitogenomes were sequenced at Berry Genomic (Beijing, China). Following quantification of the extracted total genomic DNA, an Illumina TruSeq library for a single species was obtained from the pooled genomic DNA with an average insert size of $350 \mathrm{bp}$. This library was sequenced on a full run of Illumina Hiseq 2500 with 500 cycles and paired-end sequencing (150 bp reads).

\section{Genome assembly, annotation, and analysis}

FastQC v0.11.4 (www.bioinformatics.babraham.ac.uk/projects/fastqc) was used to evaluate the quality of the raw sequences; those with an average quality value of $<$ Q30 of the putative mitochondrial genome reads were filtered out before assembly. The clean sequences were then assembled using the MitoZ v2.4 software (Meng et al., 2019) with the default parameters.

The 12 mitogenomes were initially annotated using the MITOS web server (http://mitos.bioinf.uni-leipzig.de/index.py) (Bernt et al., 2013) with invertebrate genetic codes. The locations and secondary structures of 22 tRNA genes were predicted using the MITOS WebServer and tRNAscan-SE search server (http://lowelab.ucsc.edu/tRNAscan-SE) (Schattner et al., 2005) with the extended option of invertebrate codon predictors (Lowe and Eddy, 1997). Thirteen PCGs were predicted by determining their open reading frames using the invertebrate mitochondrial genetic codons. AT-rich regions and two rRNA genes were determined based on the locations of adjacent tRNA genes and comparisons with homologous genes from other species of Caliscelidae. Mitogenomic circular maps were created and annotated using Geneious R9 (Kearse et al., 2012).

The nucleotide composition and relative synonymous codon usage (RSCU) were obtained using PhyloSuite (Zhang et al., 2020), and RSCU figures were created using the fmsb package (Bivand et al., 2019) of R 3.6.1 (R Core Team, 2019). The composition of skew was calculated according to the following formulas: AT skew $=(A-T) /(A+T) ; G C$ skew $=(G-C) /(G+C)$ (Perna and Kocher, 1995). Repeated sequences in the A+T-rich region were found using the Tandem Repeats Finder program (http://tandem.bu.edu/trf/trf.html) (Benson, 1999). The 
135

136

137

138

139

140

141

142

143

144

145

146

147

148

149

150

151

152

153

154

155

156

157

158

159

160

161

overlapping regions and intergenic spacers between genes were manually counted. The data of nucleotide diversity and the ratio of nonsynonymous substitutions $(\mathrm{Ka})$ to synonymous substitutions (Ks) for all PCGs were collected as previously described in Yang et al. (2019), specifically, calculated using DNASP v5.0 (Librado and Rozas, 2009). The sequence data of the 12 insect mitogenomes have been deposited in GenBank under the accession numbers MT577030 for Neosymplana vittatum, MW550299-MW550301 for Symplanella brevicephala, Symplana brevistrata, and Symplana lii, MW928525-MW928530 for Cylindratus longicephalus, Augilodes binghami, Pseudosymplanella nigrifasciata, Augilina triaina, Augilina tetraina, and Peltonotellus sp., and MZ343194-MZ343194 for Caliscelis shandongensis and Symplanella unipuncta, respectively.

\section{Phylogenetic analyses}

In addition to the 12 mitogenomes sequenced in this study, the complete mitogenomes of nine planthopper species were downloaded from GenBank for phylogenetic analyses; the mitogenomes of Ricania marginalis, Ricania speculum, Sivaloka damnosus, Hemisphaerius rufovarius, and Geisha distinctissima were used as outgroups. Detailed information and accession numbers of these mitogenomes are listed in Table S1.

The nucleotide sequences of 13 PCGs were aligned using MEGA v6 (Tamura et al, 2013) with Muscle (Edgar, 2004). Individual genes were concatenated using SequenceMatrix v1.7 (Vaidya et al., 2011). The optimal partition strategy and substitution models for Bayesian inference (BI) and maximum likelihood (ML) analyses were selected using the partition scheme of the software PartitionFinder v2.1.1 (Lanfear et al., 2017) with the greedy algorithm (Lanfear et al., 2012). The best-selected partitioning schemes and models for ML and BI analyses are listed in Table S2. ML analyses were performed using IQ-TREE v1.6.3 (Nguyen et al., 2014) with 10,000 replicates of ultrafast likelihood bootstrapping (Minh et al., 2013) to obtain node support values. BI analyses were conducted using MrBayes v3.2.215 (Ronquist et al., 2012) under the following conditions: two independent Markov chain Monte Carlo runs for 1,000,000 
162

163

164

165

166

167

168

169

170

171

172

173

174

175

176

177

178

179

180

181

182

183

184

185

186

187

188

generations, sampling every 1,000 generations, with a burn-in of $25 \%$. The convergence between the two runs was established by Tracer v1.6 (effective sample size > 200) (Rambaut et al., 2014).

Stationarity was considered to have been reached when the average standard deviation of split frequencies decreased to $<0.01$ and remained stable. Bootstrap percentages (BPs) of $>75 \%$ or Bayesian posterior probabilities (BPPs) of $>0.9$ were considered credible (Hillis and Bull, 1993; Mutanen et al., 2010). Finally, phylogenetic trees were viewed and edited using FigTree 1.4.2 (Mousavi et al., 2014).

\section{Results}

\section{Genome organization and composition}

The 12 newly sequenced mitogenomes were all circular double-stranded molecules with lengths ranging from 15,424 bp in Pseudosymplanella nigrifasciata to $16,746 \mathrm{bp}$ in Neosymplana vittatum (Figure 1; Table S3). Each newly sequenced mitogenome presented 37 typical metazoan mitochondrial genes, including 13 PCGs, 22 putative tRNA genes, two rRNA genes, and a large noncoding A+T-rich region (Figure 1). Among these 37 genes, 23 (9 PCGs and 14 tRNAs) were found on the major strand (J-strand), whereas the remaining 14 (4 PCGs, 2 rRNAs, and 8 tRNAs) were found on the minor strand (N-strand) (Figure 1; Table S3).

The AT nucleotide content of the 12 mitogenomes was similar: an average of $78.4 \%$ to $79.6 \%$ (Table S4). They all showed a positive AT skew ( 0.119 to 0.227 ) and a negative GC skew ( -0.298 to -0.177$)$, indicating strong AT bias, similar to that in other Caliscelidae insects [4]. The highest and lowest $\mathrm{A}+\mathrm{T}$ content was present in the control region (80.2\%-89.1\%) and PCGs (77.5\%-78.8\%), respectively (Table S4), similar to that in all previously sequenced mitogenomes of fulgoroid planthoppers (Xu et al., 2019).

\section{PCGs and codon usage}

The total length of the 13 PCGs of 12 species were ranging from 10,931 to 10,983 bp; their average AT content was $77.5 \%$ to $78.8 \%$. The AT ( $(-0.155$ to -0.122$)$ and $\mathrm{GC}(-0.088$ to -0.036$)$ 
skewness of the PCGs was similar among the 12 planthopper species (Table S4). Of the PCGs, the gene length of $n d 5$ and atp 8 was the longest and shortest, respectively (Table S3). Four of the 13 PCGs ( $n d 1, n d 4, n d 4 l$, and $n d 5)$ were coded on the minor strand, whereas the other nine (cox1, cox 2, cox3, atp6, atp $8, n d 2, n d 3, n d 6$, and $c y t b)$ were encoded on the major strand (Table S3; Figure 1).

In the 12 newly sequenced mitogenomes, most PCGs used the typical ATN as initiation codons. However, the start codon GTG was also used in $n d l$ and $n d 5$. The typical stop codon TAA occurred more frequently than TAG, and a single T was also frequently used as the stop codon. The presence of an incomplete stop codon is common in insects; it is believed to be completed by posttranscriptional polyadenylation (Ojala et al., 1981).

The codon usage pattern, RSCU, and number in the PCGs of the 12 planthopper mitogenomes were determined (Figures 2 and 3; Table S5). Analysis of PCG codon usage showed similar results among species, with Phe-UUU, Ile-AUU, Leu2-UUA, and Met-AUA being most frequently used as codons (Figure 2); these accounted for $>50 \%$ of the total number of amino acids. All were solely composed of A or $\mathrm{U}$, which is reflected in the high $\mathrm{A}+\mathrm{T}$ content of the PCGs. Augilina triaina included 59 available codons, Symplanella unipuncta included 60 available codons, Symplana brevistrata included 61 available codons, Augilina tetraina, Symplanella brevicephala, Augilodes binghami, Cylindratus longicephalus, Caliscelis shandongensis, and Peltonotellus sp. included 62 available codons, Symplana lii and Neosymplana vittatum included 63 available codons, whereas Pseudosymplanella nigrifasciata included 64 available codons (excluding TAA and TAG) (Table S5). The RSCU values of the PCGs showed a trend toward the use of A/T compared with that of G/C. Furthermore, codon usage revealed an extremely high $\mathrm{A}+\mathrm{T}$ bias that played a pivotal role in the $\mathrm{A}+\mathrm{T}$ bias of the complete mitogenome. Among the 12 newly sequenced mitogenomes, the AT and GC skews of the PCGs were negative.

\section{rRNA and tRNA genes}


216

217

218

219

220

221

222

223

224

225

226

227

228

229

230

231

232

233

234

235

236

237

238

239

240

241

242

Both small rRNA $(r r n S)$ and large rRNA $(r r n L)$ genes were found in all of the newly sequenced mitogenomes; they were located between $\operatorname{trn} V$ and the A+T-rich region and between $\operatorname{trn} V$ and $\operatorname{trnL} 1$, respectively, and both were oriented on the minor strand (Figure 1). Among the 12 mitogenomes, the length of $r r n L$ ranged from 1,205 bp in Caliscelis shandongensis to 1,238 bp in Augilodes binghami; the length of $r r n S$ ranged from 709 bp in Pseudosymplanella nigrifasciata, Symplanella brevicephala, and Symplanella unipuncta to 732 bp in Caliscelis shandongensis. For the rRNAs, the AT-skew was negative $(-0.21$ to -0.129$)$ and GC-skew was positive ( 0.256 to 0.354$)$; these genes had the second highest $\mathrm{A}+\mathrm{T}$ content in the genome (Table S4).

There was a typical set of 22 tRNAs sized 55-74 bp and interspersed throughout the mitogenome (Figure 1; Table S3). The overall length of all tRNAs was in the range of 1,4091,455 bp. Among these genes, 14 and 8 tRNAs were encoded by the major and minor strands, respectively. For all tRNAs, the AT-skew and GC-skew were positive and the A+T content was high (>77.8\%; Table S4). Of the 22 tRNAs, 2 genes (trnS1, and trnV) of Cylindratus longicephalus, Caliscelis shandongensis, and Peltonotellus sp. of the subfamily Caliscelinae lacked a DHU stem, and 3 genes ( $\operatorname{trn} C, \operatorname{trnS1}$, and $\operatorname{trn} V)$ of the remaining nine species of the subfamily Ommatidiotinae lacked a dihydrouridine arm (DHU stem) and therefore formed a simple loop, whereas the remaining could fold into canonical cloverleaf secondary structures (Figure S1-S12).

Based on the alignment of tRNAs of 12 Caliscelidae species, the percentage of identical nucleotides (\%INUC) was calculated (Table S6). The $\operatorname{trn} K$ located on the major strand had the highest \%INUC (79.45\%), whereas $\operatorname{trnC}$ on the minor strand had a \%INUC of only $33.78 \%$. Three tRNAs (trnE, trnK, and $\operatorname{trn} L 2)$ located on the major strand exhibited high levels of conservation with an average identity of $>70 \%$, indicating that tRNAs on the major strand were highly conserved. The tRNA stem was highly conserved. The base variation of the anticodon loops was low and relatively conserved, and the size of the anticodon loops was highly conserved (all $7 \mathrm{bp}$ ); the remaining loops showed different degrees of base variation, and the 
243 T $\psi C$ loops showed the most variation. Thus, the nucleotide substitutions occurred less on tRNA

244 stems than on loops, indicating higher conservation of tRNA stems.

245

246

247

248

249

250

251

252

253

254

255

256

257

258

259

260

261

262

263

264

265

266

267

268

269

\section{Overlapping and intergenic spacer regions}

The 12 mitogenomes contained 13-23 intergenic spacers, which ranged in size from 1 to 66 bp. Augilina tetraina had the longest intergenic spacer (66 bp), situated between trnH and nad4. The 12 species had 5-11 overlapping genes, with overlaps ranging in size from 1 to 8 bp (Table S3). Three gene overlaps were conserved among the 12 newly sequenced mitogenomes: $\operatorname{trn} W_{-}$ trnC (8 bp: AAGCCTTA), atp8-atp6 (7 bp: ATGATAA) except Caliscelis shandongensis, and nad4-nad4L (7 bp: TTAACAT).

\section{The AT-rich region}

The AT-rich region is involved in regulating the replication and transcription of the mitogenome in insects (Zhang and Hewitt, 1997). The control region is the largest noncoding region present between $r r n S$ and $\operatorname{trnI}$ (Figure 1). Among the 12 species, the length of the AT-rich regions ranged from $980 \mathrm{bp}$ in Symplanella brevicephala to 2,319 bp in Neosymplana vittatum. The AT-rich region contained the highest AT content of the complete mitogenome, ranging from $80.2 \%$ in Peltonotellus sp. to $89.1 \%$ in Symplanella unipuncta. The AT skewness of Augilodes binghami, Symplanella unipuncta, Symplanella brevicephala, and Symplana brevistrata was negative, indicating a pattern toward T compared with A. All species had a negative GC skew $(-0.464$ to -0.074$)$. The structural organization of the AT-rich regions in the 12 planthopper mitogenomes is illustrated in Figures S13-S24. Four repeat regions were present in Neosymplana vittatum, two were present in Peltonotellus sp., Cylindratus longicephalus, Augilodes binghami, Pseudosymplanella nigrifasciata, Symplanella unipuncta, Symplanella brevicephala, Symplana lii, and Symplana brevistrata, whereas one repeat region was present in Caliscelis shandongensis, Augilina tetraina, and Augilina triaina. The largest repeat unit, with two repeats in Augilina triaina, was $186 \mathrm{bp}$ in length. 


\section{Phylogenetic relationships}

272

273

274

275

276

277

278

279

280

281

282

283

284

285

286

287

288

289

290

291

292

293

294

295

296

All 21 species from Fulgoroidea (including the 12 species with newly sequenced mitogenomes, four Caliscelidae species were downloaded from GenBank and five outgroup species) were subjected to phylogenetic analysis based on the concatenated nucleotide sequences of 13 PCGs; using ML and BI analyses, two phylogenetic trees were obtained. These two trees had the most consistent topologies and higher node support values; thus, they were merged (Figure 4). In the consent tree, all species of Caliscelidae formed a steadily monophyletic group with high support $(\mathrm{BPPs}=1 ; \mathrm{BPs}=100)$. At the genus level, $($ Peltonotellus $+\{$ Caliscelis + [Cylindratus + Bambusicaliscelis $]\})$ formed one clade with high support $(\mathrm{BPPs}=1 ; \mathrm{BPs}=100)$, while (Youtuus $+\{$ Augilodes $+[($ Symplanella $+<$ Pseudosymplanella + Neosymplana $>)+$ $<$ Symplana + Augilina $>]\})$ formed one clade with high support (BPPs $=1$; BPs $>99)$. At the tribe level, Augilini formed sister group with the clade containing Peltonotellini and Caliscelini, Caliscelini was the sister group to Peltonotellini. At the subfamily level, Caliscelinae was the sister group to Ommatidiotinae with high nodal support $(\mathrm{BPPs}=1 ; \mathrm{BPs}=100)$.

\section{Nucleotide diversity and evolutionary rate analysis}

To investigate the evolutionary rates of the mitochondrial PCGs, nucleotide diversity, Ka, $\mathrm{Ks}$, and the $\mathrm{Ka} / \mathrm{Ks}$ ratio were calculated across 16 mitogenomes of Caliscelidae for each aligned PCG (Figure 5; Table S7) (Nei and Gojobori, 1986). Nucleotide diversity values for the individual genes ranged from 0.164 (coxl) to 0.338 (atp8). Other genes with comparatively low nucleotide diversity values included coxl (0.164), nadl (0.177) and cytb (0.177), whereas those with comparatively high values included $\operatorname{atp} 8(0.338)$, nad6 (0.271), and nad2 (0.266). The $\mathrm{Ka} / \mathrm{Ks}$ substitution ratio can be used to estimate whether a sequence is undergoing purifying, neutral, or positive selection. In pairwise $\mathrm{Ka} / \mathrm{Ks}$ analyses of the 16 sequenced mitogenomes, average $\mathrm{Ka} / \mathrm{Ks}$ ratios ranged from 0.093 for $\operatorname{cox} 1$ to 0.735 for atp 8 ; all ratios were consistently $<1$, indicating that all PCGs from the Caliscelidae mitogenomes were evolving under purifying 
297

298

299

300

301

302

303

304

305

306

selection. From these analyses, it can be concluded that $\operatorname{cox} l$ exhibited the strongest purifying selection, whereas the nad family genes exhibited a slightly relaxed purifying selection. In addition, atp 8 exhibited the least selection pressure and the fastest evolution rate among the mitochondrial PCGs of Caliscelidae, consistent with the findings of previous research (Yang et al., 2019).

\section{Discussion}

Using next-generation sequencing, we successfully sequenced and then analyzed 12 complete mitochondrial genomes of species from Caliscelidae. To the best of our knowledge, these mitogenomes add to only four other complete mitochondrial gene sequences in the NCBI database, which does not sufficiently represent $>240$ known species of Caliscelidae. Compared with the lengths of the four previously sequenced caliscelid sequences $(15,922$ bp in Youtuus erythrus to 16,640 bp in Youtuus strigatus) (Gong et al., 2021a), the 12 sequences obtained here did not show high variation in length $(15,424-16,746 \mathrm{bp})$. However, the sequences reported here lie between the 14,367 and 17,619 bp of Nilaparvata lugens from other known Fulgoroidea mitogenomes (Zhang et al., 2013; Lv et al., 2015). These differences in mitogenome size can mainly be attributed to variations in the A+T-rich region and more tandem repeats (Wang et al., 2019). Previously, the presence of gene rearrangement has only been reported in Delphacidae mitogenomes among all the Fulgoroidea insects reported to date; compared with the putative ancestral gene order, the positions of five tRNA genes $(\operatorname{trn} C, \operatorname{trn} W, \operatorname{trn} H, \operatorname{trn} P$, and $\operatorname{trn} T)$ and three PCGs (nad4, nad4l, and nad6) are inverted or translocated (Zhang et al., 2013; Lv et al., 2015; Song and Liang, 2009). However, none of the 12 newly sequenced caliscelid mitogenomes had any gene rearrangement. Indeed, the gene order and orientation of the mitogenomes of the 12 planthoppers were identical to those of the published mitogenomes of Caliscelidae, consistent with ancestral arthropod mitogenomes (Boore, 1999; Xu et al., 2019; Huang and Qin, 2018). Except for nad6 (29 bp) and cytb (21 bp), gene length differences did not exceed $20 \mathrm{bp}$; nad2 was of the exact same length, indicating that the PCGs have relatively conserved features. 
324 Furthermore, these results are consistent with those from a previous study (Xu et al., 2019). The

325 location, length, and AT content of rRNAs were highly conserved in the Caliscelidae species

326 sequenced here, similar to those of previously sequenced planthoppers (Gong et al., 2021a; Xu et

327 al., 2019; Song and Liang, 2009).

328 Among the 22 putative tRNA secondary structures, 2 genes (trnS1, and trn $V$ ) of three species of the subfamily Caliscelinae lacked a DHU stem and 3 genes (trnC, $\operatorname{trnS} 1$, and $\operatorname{trn} V)$ of remaining nine of subfamily Ommatidiotinae lacked a DHU stem, and therefore formed a simple loop. The DHU arm of trnS1 also formed a simple loop, similar to that in several other hemipterans (Song and Liang, 2009; Lee et al., 2009; Hua et al., 2009; Li et al., 2011) and metazoans (Lavrov et al., 2000). In addition, the lack of a DHU stem in $t r n V$ has been reported in Caliscelidae as well as in Aphaena discolor and A. amabilis of Fulgoridae (Wang et al., 2019). The DHU arm of $\operatorname{trn} C$ formed a simple loop, which has also been reported in Ommatidiotinae of Caliscelidae, whereas $\operatorname{trnC}$ in Caliscelinae of Caliscelidae is normal (Gong et al., 2021); these differences are species-specific. Furthermore, $\operatorname{trn} G$ and $\operatorname{trnS} 2$ in Sogatella furcifera and trnH in Laodelphax striatellus of Delphacidae cannot form a typical cloverleaf structure (Song and Liang, 2009; Zhang et al., 2014; Yu and Liang, 2018). In the present study, the percentage of identical nucleotides showed that conservation of tRNAs was high on the major strand, which is consistent with previous findings (Zhang et al., 2019). The anticodon arm and loops were the most conserved regions of the tRNAs, but the tRNA stems were always more conserved than the loops, which is in agreement with the findings of previous studies (Xu et al., 2019; Zhang et al., 2019).

In several Fulgoroidea species, an 8-bp overlap of "AAGCCTTA" was detected between $\operatorname{trn} W$ and $\operatorname{trn} C$. This characteristic is consistent with other Caliscelidae species (Gong et al., 2021a; Xu et al., 2019). In addition, we detected a 7-bp overlap "ATGATAA" between atp8 and atp6 in the 11 new sequenced studied species; however, in the reported mitogenomes of Bambusicaliscelis, the overlap is "ATAATAA." Therefore, perhaps owing to differences within the subfamily, species-specific A/G differences exist. A 7-bp overlap "TTAACAT" was 
351 previously found between $n d 4$ and $n d 4 l$ in Caliscelidae, but it was not found in other Fulgoroidea 352 mitogenomes (Gong et al., 2021a; Xu et al., 2019).

353 In this study, a total of 11 representative genera of the Chinese Caliscelidae family were

354

355

356

357

358

359

360

361

362

363

364

365

366

367

368

369

370

371

372

373

374

375

376

377

selected for phylogenetic analysis to understand the genetic relationship among each genus. The phylogenetic trees generated by BI and ML methods were fully resolved with identical topologies (Figure 4). Among them, Peltonotellus (Peltonotellini) is located at the middle of the evolutionary tree with three genera (Bambusicaliscelis, Cylindratus, and Caliscelis) of the tribe Caliscelini, these two tribes are grouped into the subfamily Caliscelinae, indicating a relationship similar to the previous results obtained based on morphology. Emeljanov erected the tribe Peltonotellini and the recent modern classification of the family Caliscelidae, including the tribe Peltonotellini was published by Gnezdilov (Emeljanov, 2008; Gnezdilov and Wilson, 2011; Gnezdilov, 2013). Augilini formed sister-group with the clade containing Peltonotellini and Caliscelini, Yotuus was basal to the Augilini. Augilina and Symplana are sister groups, which are so similar morphologically that they can easily be mistaken for the same genus and can only be identified by their genitalia (Zhang et al., 2020), phylogenetic analysis of the mitochondrial genomes of these two genera is consistent with the conclusion of morphological studies by Gong et al. indicating that Augilina and Symplana should be two genera rather than the same genus (Gong et al., 2021b). At present, the monophyly of Caliscelidae is yet to be tested because of the limited number of available mitogenomes; currently, mitochondrial data does not exist for Ommatidiotini, and Adenissini in the NCBI database. Thus, more detailed investigation is required to test the monophyly of Caliscelidae. In addition, increasing the number of mitogenome datasets might improve the reliability and robustness of phylogenetic analyses for Fulgoroidea.

The complete mitogenomes of the 12 planthoppers presented here along with the reported phylogenetic relationships may serve as a baseline dataset for future studies on population genetics. Moreover, our study improves the current understanding of mitogenome structure to develop effective diagnostic and management strategies for insect pests. 


\section{Conclusions}

In the present study, we determined and comparatively analyzed the complete mitogenomes

of 12 Caliscelidae species, namely, Augilina tetraina, Augilina triaina, Symplana brevistrata,

Symplana lii, Neosymplana vittatum, Pseudosymplanella nigrifasciata, Symplanella

brevicephala, Symplanella unipuncta, Augilodes binghami, Cylindratus longicephalus, Caliscelis

shandongensis, and Peltonotellus sp., for the first time. The 12 mitogenomes ranged from 15,424

to $16,746 \mathrm{bp}$ in length, with the typical gene content and similar arrangement of genes usually

observed in Hexapods. Among 13 PCGs in 16 reported Caliscelidae mitogenomes, coxl and atp 8

showed the lowest and highest nucleotide diversity, respectively. All PCGs evolved under

purifying selection, with atp 8 considered a comparatively fast-evolving gene. The complete

mitogenomes of the 12 planthoppers presented here along with the reported phylogenetic

relationships may serve as a baseline dataset for future studies on population genetics. Moreover,

our study improves the current understanding of mitogenome structure to develop effective

diagnostic and management strategies for insect pests.

Funding: This work was supported by the National Natural Science Foundation of China (grant nos. 32060343, 31472033); the Science and Technology Support Program of Guizhou Province

(grant no. 20201Y129); the Program of Excellent Innovation Talents, Guizhou Province (grant

no. 20154021). 
399

400

401

402

403

404

405

406

407

408

409

410

411

412

413

414

415

416

417

418

419

\section{Grant Disclosures}

The following grant information was disclosed by the authors:

National Natural Science Foundation of China: 32060343 and 31472033.

Science and Technology Support Program of Guizhou Province: 20201 Y129.

Program of Excellent Innovation Talents, Guizhou Province: 20154021.

\section{Competing Interests}

The authors declare that they have no competing interests.

\section{Author Contributions}

Nian Gong: Investigation, Formal analysis, Writing - original draft.

Lin Yang: Resources, Investigation, Formal analysis.

Xiang-Sheng Chen: Validation, Project administration, Funding acquisition.

\section{Data Availability}

The following information was supplied regarding data availability:

The sequence data of the 12 Caliscelidae mitogenomes are available at GenBank:

MT577030 for Neosymplana vittatum, MW550299-MW550301 for Symplanella brevicephala, Symplana brevistrata, and Symplana lii, MW928525-MW928530 for Cylindratus longicephalus, Augilodes binghami, Pseudosymplanella nigrifasciata, Augilina triaina, Augilina tetraina, and Peltonotellus sp., and MZ343194-MZ343194 for Caliscelis shandongensis and Symplanella unipuncta, respectively. 
References

Benson G. 1999. Tandem repeats fnder: a program to analyze DNA sequences. Nucleic Acids Research 27(2):573-580.

Bernt M, Donath A, Jühling F, Externbrink F, Florentz C, Fritzsch G, Pütz J, Middendorf M, Stadler PF. 2013. MITOS: improved de novo metazoan mitochondrial genome annotation. Molecular Phylogenetics and Evolution 69(2):313-319 DOI 10.1016/j.ympev.2012.08.023.

Bivand R, Altman M, Anselin L, Assunção R, Berke O, Bernat A. 2019. spdep: spatial dependence: weighting schemes, statistics and models. R package version 1.1-2.

Boore JL. 1999. Animal mitochondrial genomes. Nucleic Acids Research 27:1767-1780.

Bourgoin T. 2021. FLOW (Fulgoromorpha Lists on The Web): a world knowledge base dedicated to Fulgoromorpha. Version 8, updated 7 January Available from http://hemipteradatabases.org/ flow/ [accessed 7 January 2021].

Cameron SL. 2014. Insect mitochondrial genomics: implications for evolution and phylogeny. Annual Review of Entomology 59(1):95-117 DOI 10.1146/annurev-ento-011613-162007.

Che Y-L, Zhang Y-L, Webb MD. 2009. A new genus and species of the planthopper tribe Augilini Baker (Hemiptera, Caliscelidae, Ommatidiotinae) from Thailand and China. Zootaxa 2311:49-54.

Chen X-S, Zhang Z-G, Chang Z-M. 2014. Issidae and Caliscelidae (Hemiptera: Fulgoroidea) from China. Guizhou Science and Technology Publishing House, Guiyang. pp. 156-183.

Dowton M, Castro LR, Austin AD. 2002. Mitochondrial gene rearrangements as phylogenetic characters in the invertebrates: the examination of genome 'morphology'. Invertebrate Systematics 16:345-356.

Edgar RC. 2004. MUSCLE: multiple sequence alignment with high accuracy and high throughput. Nucleic Acids Research 32(5):1792-1797 DOI 10.1093/nar/gkh340.

Emeljanov A. F. 2008. New species of the genus Peltonotellus Puton (Homoptera, Caliscelidae) from Kazakhstan, Middle, and Central Asia. Tethys Entomological Research 16:5-12.

Gissi C, Iannelli F, Pesole G. 2008. Evolution of the mitochondrial genome of Metazoa as exemplified by comparison of congeneric species. Heredity 101:301-320.

Gnezdilov V.M., Wilson M.R. 2011. Order Hemiptera, family Caliscelidae. Arthropod fauna of the UAE. 4:114-122.

Gnezdilov V.M. 2013. A Modern Classification of the Family Caliscelidae Amyot et Serville (Homoptera, Fulgoroidea). Zoologicheskii Zhurnal. 92 (11):1309-1311. [English translation published in Entomological Review. 2014, 94 (2):211-214].

Gong N, Yang L, Chen X-S. 2018. Youtuus, a new bamboo-feeding genus of the tribe Augilini with two new species from China (Hemiptera, Fulgoromorpha, Caliscelidae). ZooKeys 783:85-96 DOI 10.3897/ zookeys.783.25135.

Gong N, Yang L, Chen X-S. 2020. Two new species of the genus symplanella fennah 
(Hemiptera, Fulgoromorpha, Caliscelidae) from china. Zootaxa 4801(2):355-362.

Gong N, Yang L, Chen X-S. 2021a. Structural features and phylogenetic implications of four new mitogenomes of Caliscelidae (Hemiptera: Fulgoromorpha). International Journal of Molecular Sciences 22:1348 DOI 10.3390/ijms22031348

Gong N., Yang L., Chen X.-S. 2021b. First record of the genus Augilina Melichar, 1914 (Hemiptera, Fulgoromorpha, Caliscelidae) from China, with descriptions of two new bamboo-feeding species. European Journal of Taxonomy 744:38-48. DOI 10.5852/ejt.2021.744.1297

Gwiazdowski RA, Foottit RG, Maw HEL, Hebert PDN. 2015. The Hemiptera (Insecta) of Canada: constructing a reference library of DNA barcodes. Plos One 10(4): e0125635-.

Hebert PDN, Ratnasingham S, Zakharov EV, Telfer AC, Valerie LB, Milton MA, Pedersen S, Jannetta P, Jeremy R de W. 2016. Counting animal species with DNA barcodes: Canadian insects. Philosophical Transactions of the Royal Society B-Biological Sciences 371(1702):20150333.

Hillis DM, Bull JJ. 1993. An empirical test of bootstrapping as a method for assessing confidence in phylogenetic analysis. Systematic Biology 42 (2):182-192.

Hua J-M, Li M, Dong P-Z, Cui Y, Xie Q, Bu W-J. 2009. Phylogenetic analysis of the true water bugs (Insecta: Hemiptera: Heteroptera: Nepomorpha): evidence from mitochondrial genomes. BMC Evolutionary Biology 9:134.

Huang Y-X, Qin D-Z. 2018. Sequencing and analysis of the complete mitochondrial genome of Changeondelphax velitchkovskyi (Hemiptera: Fulgoroidea). Mitochondrial DNA Part B 3:1, 90-91 DOI 10.1080/23802359.2017.1422407.

Ingman M, Kaessmann H, Paabo S, Gyllensten U. 2000. Mitochondrial genome variation and the origin of modern humans. Nature 408:708-713.

Inohira K, Hara T, Matsuura ET. 1997. Nucleotide sequence divergence in the A+T-rich region of mitochondrial DNA in Drosophila simulans and Drosophila mauritiana. Molecular Biology and Evolution 14(8):814-822 DOI 10.1093/oxfordjournals.molbev.a025822.

Kearse M, Moir R, Wilson A, Stoneshavas S, Cheung M, Sturrock S, Buxton S, Cooper A, Markowitz S, Duran C. 2012. Geneious Basic: an integrated and extendable desktop software platform for the organization and analysis of sequence data. Bioinformatics 28 (12):1647-1649.

Lanfear R, Calcott B, Ho SY, Guindon S. 2012. PartitionFinder: combined selection of partitioning schemes and substitution models for phylogenetic analyses. Molecular biology and evolution 29(6):1695-1701.

Lanfear R, Frandsen PB, Wright AM, Senfeld T, Calcott B. 2017. PartitionFinder 2: new methods for selecting partitioned models of evolution for molecular and morphological phylogenetic analyses. Molecular Biology and Evolution 34(3):772-773 DOI 10.1093/molbev/msw260.

Lavrov D-V, Brown W-M, Boore J-L. 2000. A novel type of RNA editing occurs in the mitochondrial tRNAs of the centipede Lithobius forficatus. Proceedings of the National 
500

501

502

503

504

505

506

507

508

509

510

511

512

513

514

515

516

517

518

519

520

521

522

523

524

525

526

527

528

529

530

531

532

533

534

535

536

537

538

539

540

Academy of Sciences of the United States of America 97(25):13738-13742

Lee W, Kang J, Jung C, Hoelmer K, Lee SH, Lee S. 2009. Complete mitochondrial genome of brown marmorated stink bug Halyomorpha halys (Hemiptera: Pentatomidae), and phylogenetic relationships of hemipteran suborders. Molecules and Cells 28(3):155-165

Li H, Gao J-Y, Liu H-Y, Liu H, Liang A-P, Zhou X-G, Cai W-Z. 2011. The architecture and complete sequence of mitochondrial genome of an assassin bug Agriosphodrus Dohrni (Hemiptera: Reduviidae). International Journal of Biological Sciences 7(6):792-804.

Librado P, Rozas J. 2009. DnaSP v5: a software for comprehensive analysis of DNA polymorphism data. Bioinformatics 25:1451-1452.

Lowe TM, Eddy SR. 1997. tRNAscan-SE: a program for improved detection of transfer RNA genes in genomic sequence. Nucleic Acids Research 25(5):955-964 DOI 10.1093/nar/25.5.955.

Lv L, Peng X-X, Jing S-L, Liu B-F, Zhu L-L, Hei G-C. 2015. Intraspecific and interspecific variations in the mitochondrial genomes of Nilaparvata (Hemiptera: Delphacidae). Journal of Economic Entomology 4:2021-9.

Ma C, Yang P, Jiang F, Chapuis MP, Shali Y, Sword GA, Kang L. 2012. Mitochondrial genomes reveal the global phylogeography and dispersal routes of the migratory locust. Molecular Ecology 21:4344-4358.

Meng G, Li Y, Yang C, Liu S. 2019. MitoZ: a toolkit for animal mitochondrial genome assembly, annotation and visualization. Nucleic Acids Research 47(11): e63-e63.

Meng R, Gnezdilov V.M., Wang Y. 2015. Two new species of the genus Peltonotellus Puton (Hemiptera: Fulgoromorpha: Caliscelidae) from northwestern China with a world checklist. Zootaxa 4052(4): 465.

Minh BQ, Nquyen MA, von Haeseler A. 2013. Ultrafast approximation for phylogenetic bootstrap. Molecular Biology and Evolution 30(5):1188-1195 DOI 10.1093/molbev/mst024.

Mousavi SA, Österman J, Wahlberg N, Nesme X, Lavire C, Vial L, Paulin L, de Lajudie P, Lindström K. 2014. Phylogeny of the Rhizobium-Allorhizobium-Agrobacterium clade supports the delineation of Neorhizobium gen. nov. Systematic and Applied Microbiology 37:208-215

Mutanen M, Wahlberg N, Kaila L. 2010. Comprehensive gene and taxon coverage elucidates radiation patterns in moths and butterflies. Proceedings Biological Sciences 277 (1695):2839-2848.

Nei M, Gojobori T. 1986. Simple methods for estimating the numbers of synonymous and nonsynonymous nucleotide substitutions. Molecular Biology and Evolution 3:418-426 DOI 10.1093/oxfordjournals.molbev.a040410.

Nguyen LT, Schmidt HA, von Haeseler A, Minh BQ. 2014. IQ-TREE: a fast and efective stochastic algorithm for estimating maximum-likelihood phylogenies. Molecular Biology and Evolution 32(1):268-274.

Ojala D, Montoya J, Attardi G. 1981. tRNA punctuation model of RNA processing inhuman mitochondrial. Nature 290:470-474. 
541 Osigus HJ, Eitel M, Bernt M, Donath A, Schierwater B. 2013. Mitogenomics at the base of

542

543

544

545

546 Metazoa. Molecular Phylogenetics and Evolution 69(2):339-351.

Perna NT, Kocher TD. 1995. Patterns of nucleotide composition at fourfold degenerate sites of animal mitochondrial genomes. Journal of Molecular Evolution 41(3):353-358 DOI 10.1007/bf01215182.

R Core Team. 2019. R: A language and environment for statistical computing. R Foundation for Statistical Computing, Vienna, Austria.

Rambaut A, Suchard MA, Xie D, Drummond AJ. 2014. Tracer v1.6, Available from http:// tree.bio.ed.ac.uk/software/tracer/

Ronquist F, Teslenko M, van der Mark P, Ayres DL, Darling A, Höhna S, Larget B, Liu L, Suchard MA, Huelsenbeck JP. 2012. MrBayes 3.2: efficient Bayesian phylogenetic inference and model choice across a large model space. Systematic Biology 61:539-542

Schattner P, Brooks AN, Lowe TM. 2005. The tRNAscan-SE, snoscan and snoGPS web servers for the detection of tRNAs and snoRNAs. Nucleic Acids Research 33(Supp1_2), W686-W689.

Shadel GS, Clayton DA. 1997. Mitochondrial DNA maintenance invertebrates. Annual Review of Biochemistry 66:409-435.

Simon C, Buckley TR, Frati F, Stewart JB, Beckenbach AT. 2006. Incorporating molecular evolution into phylogenetic analysis, and a new compilation of conserved polymerase chain reaction primers for animal mitochondrial DNA. Annual Review of Ecology Evolution and Systematics 37:545-579.

Song N, Liang A-P. 2009. Complete mitochondrial genome of the small brown planthopper, Laodelphax striatellus (Delphacidae: Hemiptera), with a novel gene order. Zoological Science 26:851-860.

Tamura K, Stecher G, Peterson D, Filipski A, Kumar S. 2013. MEGA6: molecular evolutionary genetics analysis version 6.0. Molecular Biology and Evolution 30(12):27252729 DOI 10.1093/molbev/mst197.

Tian H-S, Zhu C-L, Gao X-H, Ma L, Wu G-L. 2001. Cloning and identification of deltamethrinresistance or susceptibility associated genes of culex pipiens pallens. Chinese Journal of Parasitology \& Parasitic Diseases 19(4):193-197.

Vaidya G, Lohman DJ, Meier R. 2011. SequenceMatrix: concatenation software for the fast assembly of multi-gene datasets with character set and codon information. Cladistics 27:171-180.

Wang J-J, Li D-F, Li H, Yang M-F, Dai R-H. 2019. Structural and phylogenetic implications of the complete mitochondrial genome of Ledra auditura. Scientific Reports 9:15746 DOI 10.1038/s41598-019-52337-9.

Wang W-Q, Huang Y-X, Bartlett CR, Zhou F-M, Meng R, Qin D-Z. 2019. Characterization of the complete mitochondrial genomes of two species of the genus Aphaena GuérinMéneville (Hemiptera: Fulgoridae) and its phylogenetic implications. International Journal of Biological Macromolecules 141:29-40.

Wang Q, Tang G. 2017. Genomic and phylogenetic analysis of the complete mitochondrial 

DNA sequence of walnut leaf pest, Paleosepharia Posticata, (Coleoptera: Chrysomeloidea). Journal of Asia-Pacific Entomology S1226861517301772.

Wolstenholme DR. 1992. Animal mitochondrial DNA: Structure and evolution. International Review of Cytology 141:173-216.

Xu S-Y, Long J-K, Chen X-S. 2019. Comparative analysis of the complete mitochondrial genomes of five Achilidae species (Hemiptera: Fulgoroidea) and other Fulgoroidea reveals conserved mitochondrial genome organization. Peer J 7: e6659 DOI 10.7717/peerj.6659.

Yang M, Song L, Zhou L, Shi Y, Zhang Y. 2019. Mitochondrial genomes of four satyrine butterflies and phylogenetic relationships of the family Nymphalidae (Lepidoptera: Papilionoidea). International Journal of Biological Macromolecules 145.

Yu F, Liang A-P. 2018. The Complete Mitochondrial Genome of Ugyops sp. (Hemiptera: Delphacidae). Journal of Insect Science 18(3):3.

Zhang D, Gao F, Jakovlić I, Zou H, Zhang J, Li W-X, Wang G-T. 2020. PhyloSuite: An integrated and scalable desktop platform for streamlined molecular sequence data management and evolutionary phylogenetics studies, Molecular Ecology Resources, 20(1):348-355.

Zhang DX, Hewitt GM. 1997. Insect mitochondrial control region: a review of its structure, evolution and usefulness in evolutionary studies. Biochemical Systematics and Ecology 25:99-120.

Zhang D-L, Zhang M-M, Tian J, Li Y-R, Zhang H-F. 2019. Comparative study of 22 mitochondrial Trna genes in Nepomorpha (Hemiptera: Heteroptera). Journal of Shanxi Agricultural University (Natural Science Edition) 39(01):33-40.

Zhang K-J, Zhu W-C, Rong X, Zhang Y-K, Ding X-L, Liu J, Chen D-S, Du Y, Hong X-Y. 2013. The complete mitochondrial genomes of two rice planthoppers, Nilaparvata lugens and Laodelphax striatellus: conserved genome rearrangement in delphacidae and discovery of new characteristics of atp8 and trna genes. BMC Genomics 14(1):417

Zhang K-J, Zhu W-C, Rong X, Liu J, Ding X-L, Hong X-Y. 2014. The complete mitochondrial genome sequence of Sogatella furcifera (Horváth) and a comparative mitogenomic analysis of three predominant rice planthoppers. Gene 533:100-109.

Zhang Y.-L., Che Y.-L., Meng R., Wang Y.-L. 2020. Fauna Sinica Insecta Vol. 70 (Hemiptera: Caliscelidae and Issidae). Science Press 152-153. 


\section{Figure 1}

Circular maps of the12 newly sequenced mitogenomes of Caliscelidae .

Circular maps of the12 newly sequenced mitogenomes of Caliscelidae. Protein-coding, ribosomal, and transfer RNA genes are shown with standard abbreviations. Gene orientations are indicated by arrow directions. Protein-coding genes, transfer RNA genes, control regions, and two ribosomal RNA genes are shown in yellow, aubergine, blue, and red, respectively. 

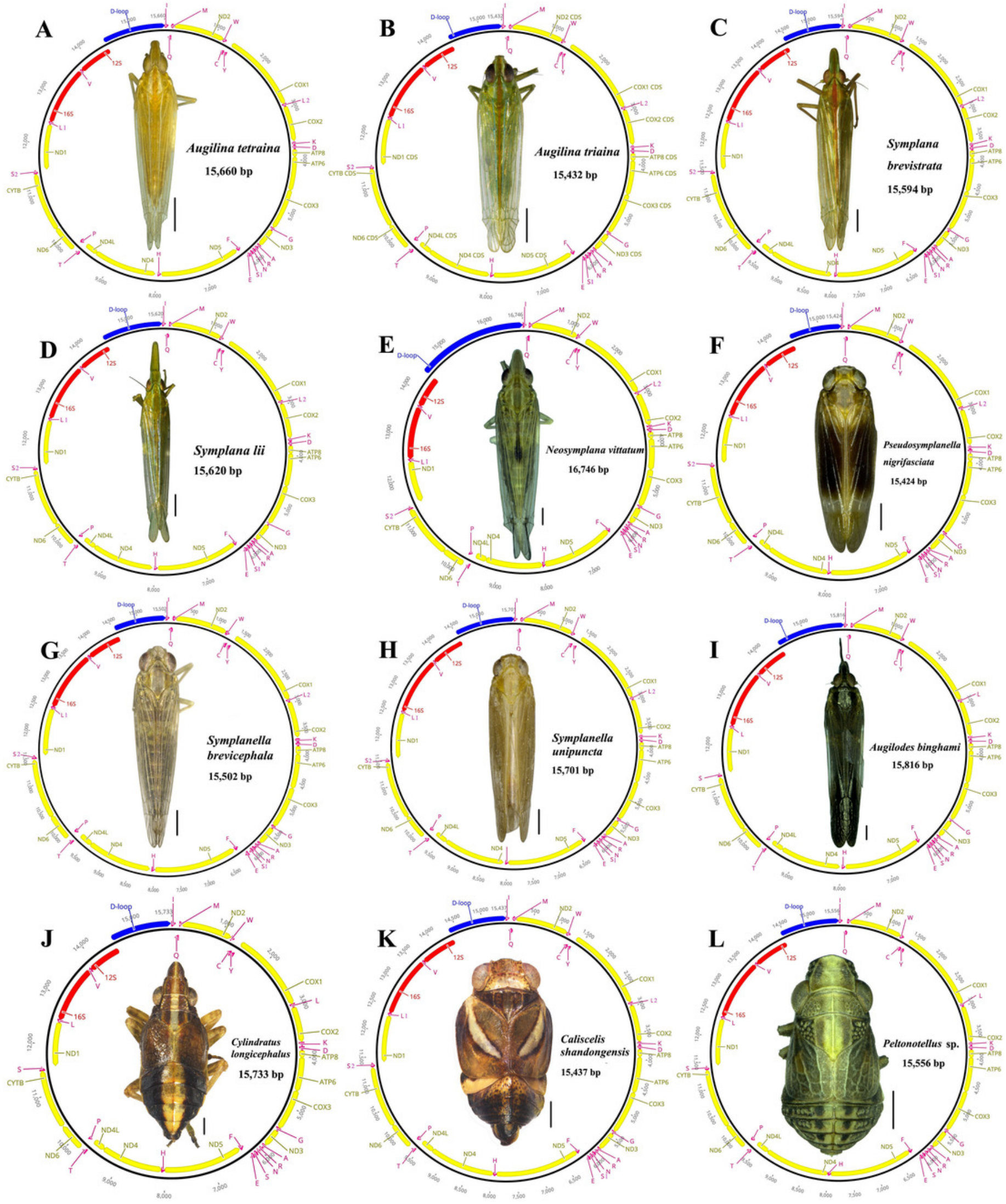
Figure 2

Number of codon usages in the protein-coding genes of the12 newly sequenced mitogenomes of Caliscelidae.

Number of codon usages in the protein-coding genes of the12 newly sequenced mitogenomes of Caliscelidae. 


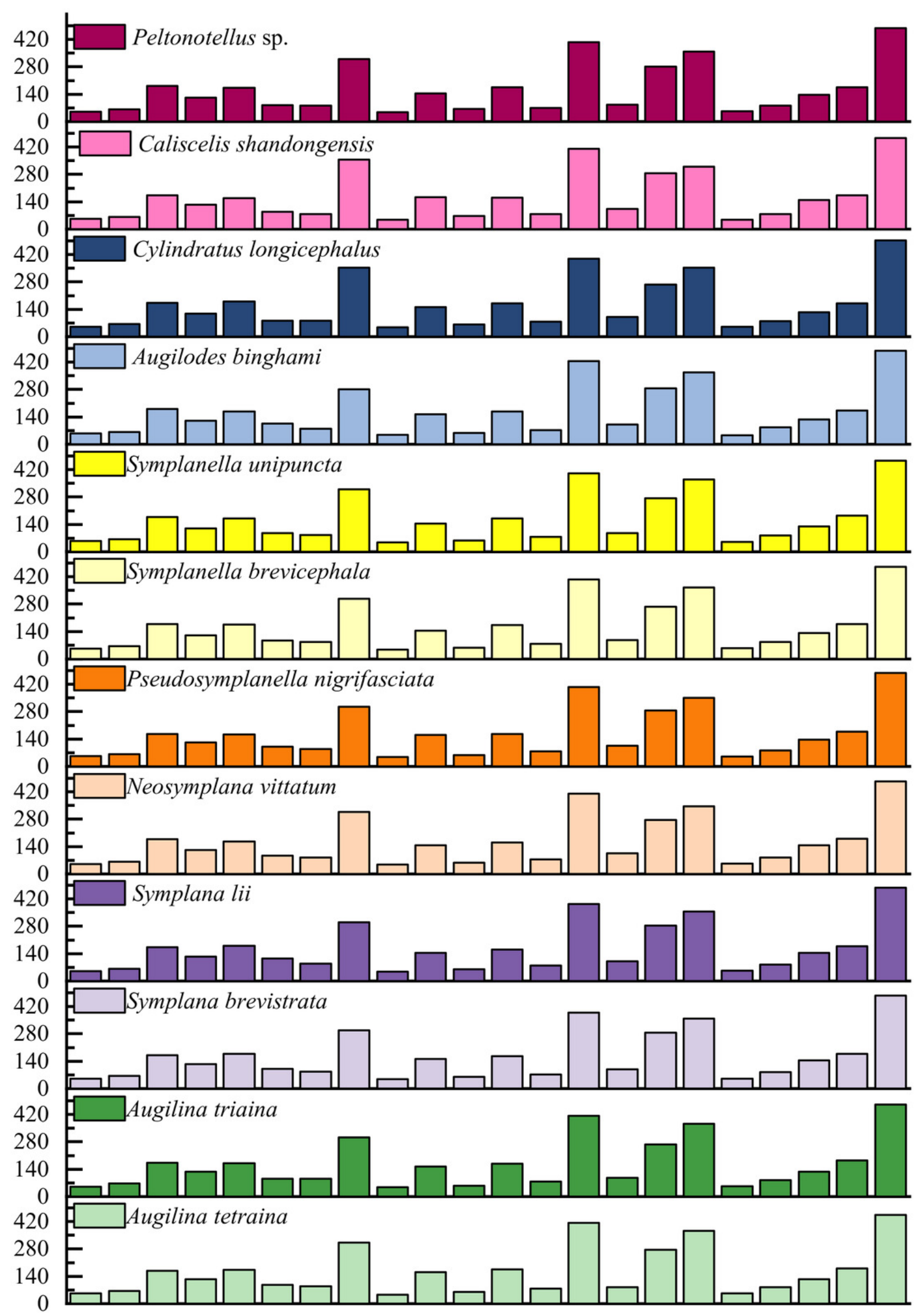

Gln His Asn Pro Thr Leu1 Glu Met Arg Tyr Asp Lys Ala Ile Ser1 Ser2Leu2 Cys Trp Val Gly Phe 


\section{Figure 3}

Relative synonymous codon usage in the protein-coding genes of the12 newly sequenced mitogenomes of Caliscelidae.

Relative synonymous codon usage in the protein-coding genes of the12 newly sequenced mitogenomes of Caliscelidae. Codon families are indicated below the X-axis. The color of the codon family below the $\mathrm{X}$-axis corresponds to the color above the $\mathrm{X}$-axis. The stop codon is not given. Codons absent in mitogenomes are shown at the top of columns. 

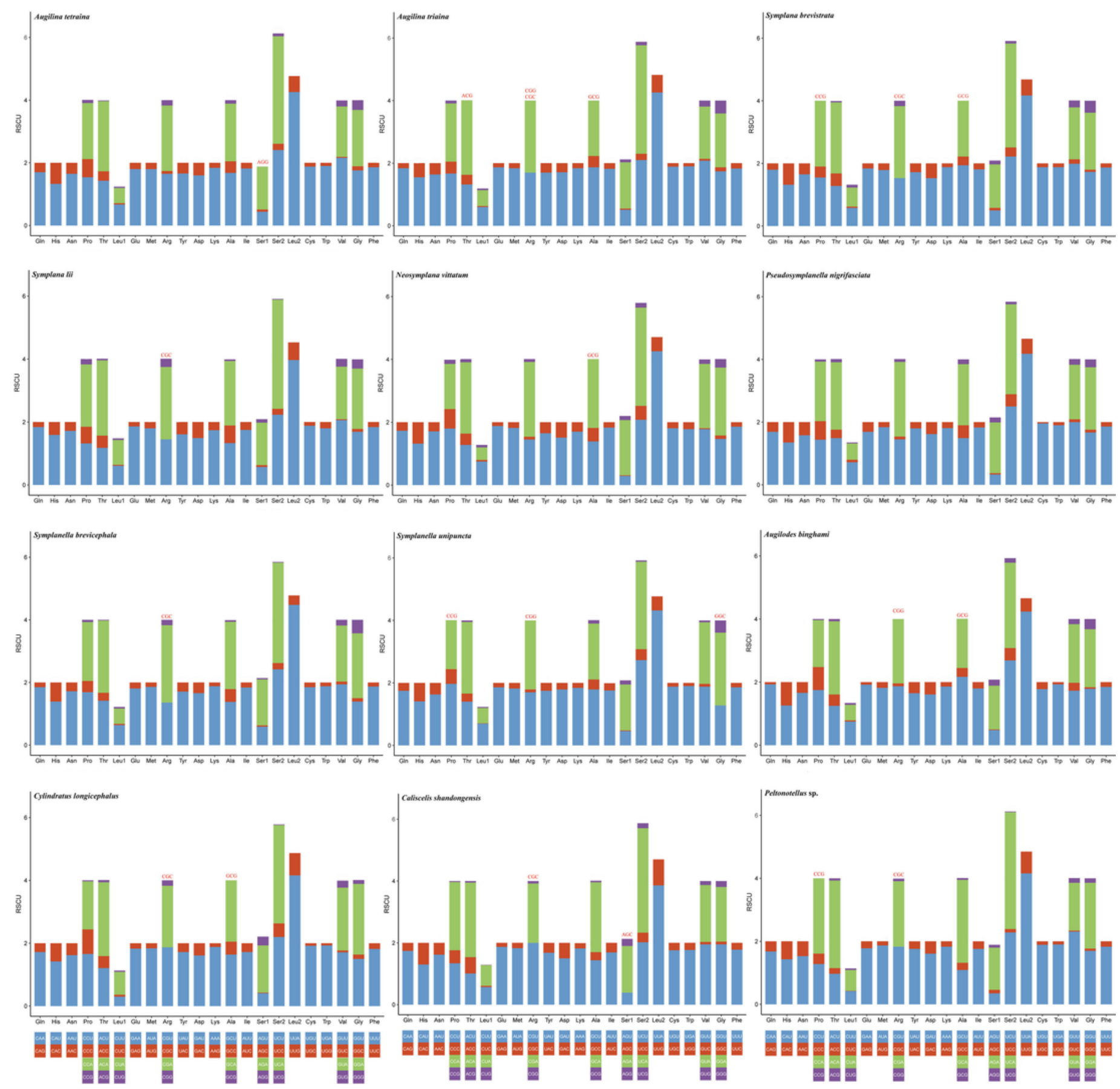
Figure 4

Phylogenetic trees of Fulgoroidea inferred using MrBayes (Bayesian inference) and maximum likelihood (ML) analysis based on the nucleotide sequences of 13 proteincoding genes.

Phylogenetic trees of Fulgoroidea inferred using MrBayes (Bayesian inference) and maximum likelihood (ML) analysis based on the nucleotide sequences of 13 protein-coding genes. Bayesian posterior probabilities (BPPs) and bootstrap percentages (BPs) are indicated on branches.

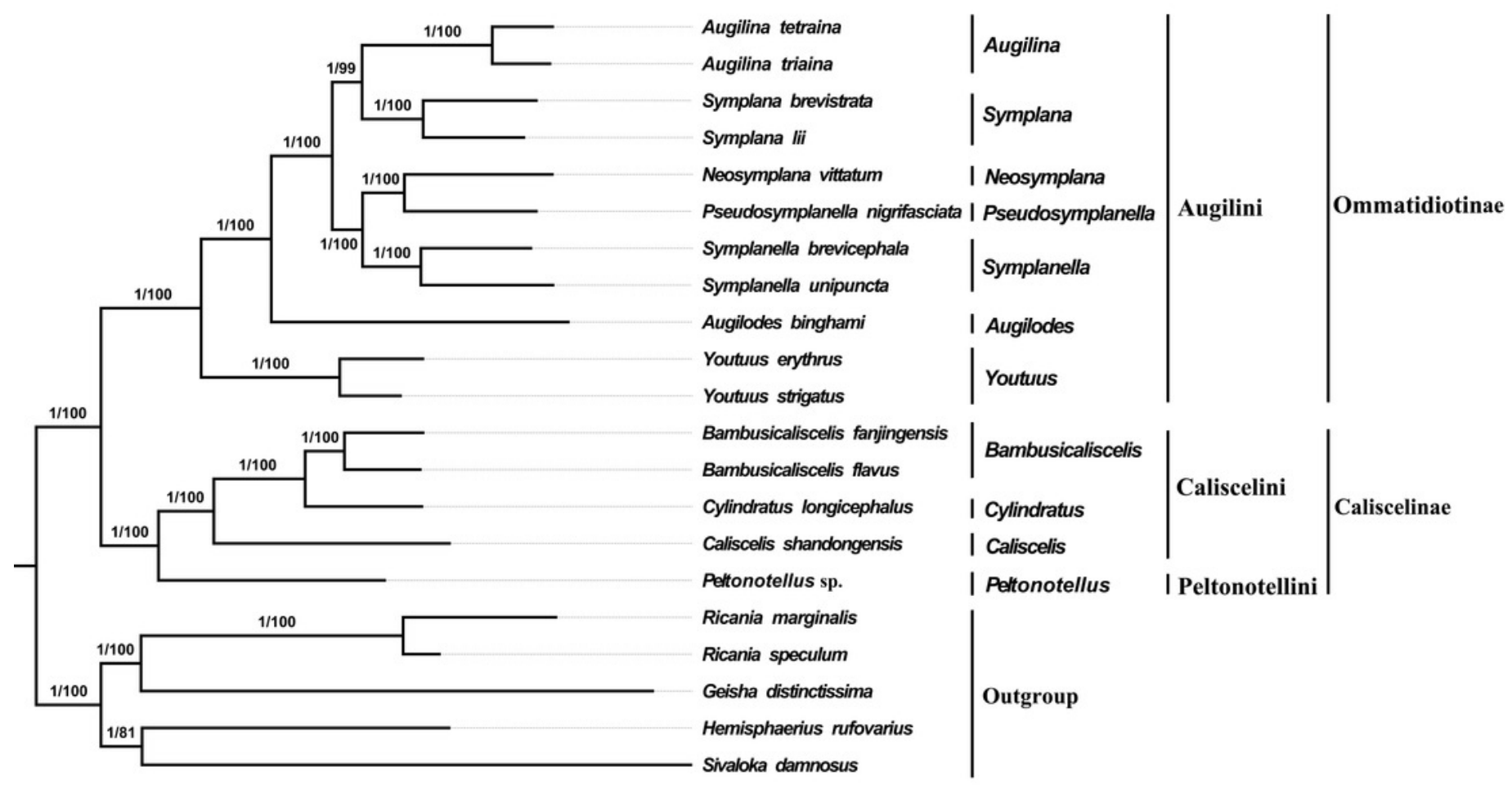


Figure 5

Nucleotide diversity (A) and the ratio of Ka/Ks (B) of protein-coding genes from 16 reported Caliscelidae mitogenomes.

Nucleotide diversity (A) and the ratio of Ka/Ks (B) of protein-coding genes from 16 reported Caliscelidae mitogenomes. 

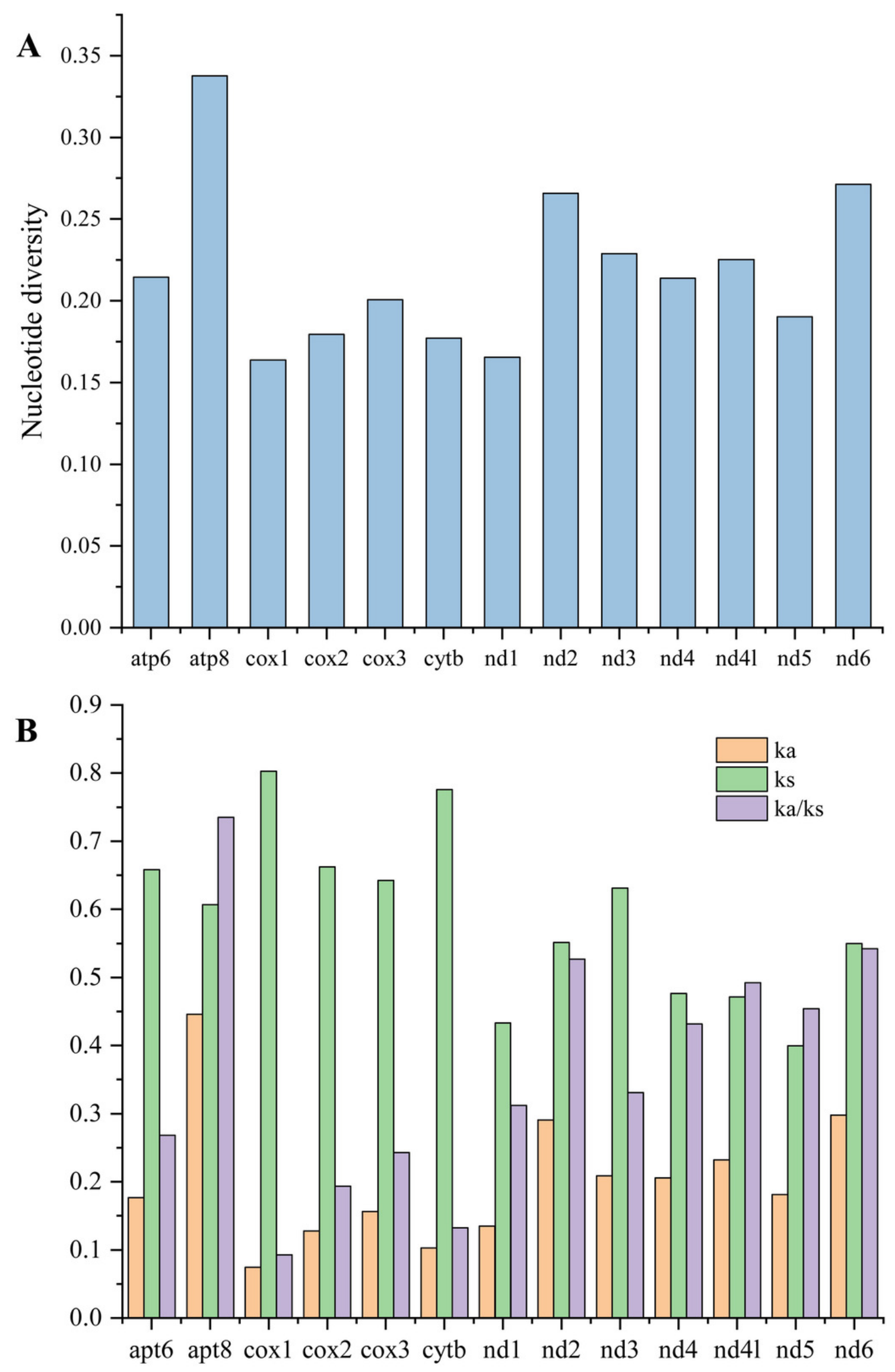